\title{
The shooting approach to optimal control problems *
}

\author{
J. Frédéric Bonnans* \\ * INRIA-Saclay and Centre de Mathématiques Appliquées, \\ Ecole Polytechnique, 91128 Palaiseau, France \\ (e-mail: Frederic.Bonnans@inria.fr).
}

\begin{abstract}
We give an overview of the shooting technique for solving deterministic optimal control problems. This approach allows to reduce locally these problems to a finite dimensional equation. We first recall the basic idea, in the case of unconstrained or control constrained problems, and show the link with second-order optimality conditions and the analysis or discretization errors. Then we focus on two cases that are now better undestood: state constrained problems, and affine control systems. We end by discussing extensions to the optimal control of a parabolic equation.
\end{abstract}

Keywords: Optimal control, singular control, state variables, state constraints, shooting algorithm, parabolic equation.

\section{INTRODUCTION, EXAMPLES}

This paper presents some recent progress in the theoretical analysis of shooting algorithms for solving (deterministic) optimal control problems of ODEs. In the case of an unconstrained optimal control problem with $n$ states, this method allows to reduce the resolution of the optimality conditions to $n$ nonlinear equations. Of course the evaluation of these equations and of their Jacobian (which is required for solving them, say, by a Newton type method) needs to integrate a differential system. Using high order integrators, one can compute an approximation of the solution with error at most $\varepsilon>0$, with computational cost $o(\varepsilon)$. This is much cheaper than the direct method, used by software packages as in Bonnans et al. [2012], which consists in solving the discretized problem by a nonlinear programming solver. However, the latter have "globalization features" that allow them (sometimes) to converge even if the starting point is poor, taking advantage of descent properties. Shooting algorithms presently need to be initialized with good starting points, typically obtained by solving the problem with a more robust approach (like dynamic programming, see e.g. the Appendix by M. Falcone in Bardi and Capuzzo-Dolcetta [1997], or by a direct approach, sometimes itself initialized with a simplified model).

This is reminiscent of the gradient algorithm introduced in Cauchy [1847], who motivated it as a way to compute a good starting point in order to apply Newton's method for

\footnotetext{
* Preliminary version, Proc. IFAC ALCOSP Conference, Caen, July 3-5, 2013, to appear. This paper benefited from collaborations and discussions with Xavier Dupuis, Audrey Hermant, Kazimierz Malanowski, Helmut Maurer, Laurent Pfeiffer, for the study of state constrained problems, and with Maria Soledad Aronna, Bean-San Goh, Andrei Dmitruk, Pierre Martinon, Pablo Lotito and Emmanuel Trélat for the study of problems with singular arcs, as well as with Francisco Silva and Nikolai Osmolovskiŭ on other aspects of optimal control problems.
}

solving nonlinear equations. He suggested then to solve the associated least-square problem by the gradient algorithm.

In the recent years progress has been made in two important cases: (i) problems with state constraints, and (ii) problems with singular arcs. In both cases the challenge was to get a correct formulation of the shooting equations, and to connect them to the second-order optimality conditions, in order to obtain locally quadratically convergent algorithms.

\section{WHAT IS THE SHOOTING ALGORITHM ?}

\subsection{Basic shooting}

Given a $C^{\infty}$ and Lipschitz vector field $f: \mathbb{R}^{n} \rightarrow \mathbb{R}^{n}$, and $T>0$, denote by $F_{T}$ the associated flow: $F_{T}(x)=y_{T}$, where $y_{t}$ is solution of $\dot{y}_{t}=f\left(y_{t}\right)$ for all $t>0$, and $y_{0}=x$. The derivative of this $C^{\infty}$ mapping is $D F_{T}(x)=Z_{T}$, where $Z_{t}, t \in(0, T)$, is a $n \times n$ matrix, solution of the linearized equation $\dot{Z}_{t}=D f\left(y_{t}\right) Z_{t}$, with initial condition $Z_{0}=I$ (identity).

Assume that, instead of a Cauchy problem, we have to solve a two-point boundary value problem of the form $\Phi\left(y_{0}, y_{T}\right)=0$, with $\Phi$ smooth (i.e., of class $\left.C^{\infty}\right) \mathbb{R}^{n} \times$ $\mathbb{R}^{n} \rightarrow \mathbb{R}^{n}$. We can reformulate this problem as $\mathcal{F}(x):=$ $\Phi\left(x, F_{T}(x)\right)=0$. A zero $\bar{x}$ of $\mathcal{F}$ (such that $\mathcal{F}(\bar{x})=0$ ) is said to be regular if $D \mathcal{F}(\bar{x})$ is invertible. In that case (actually, if $D \mathcal{F}(\bar{x})$ is sufficiently well conditioned) Newton's method is a method of choice for computing $\bar{x}$, provided we can initialize it by an initial point, close enough to $\bar{x}$. Note that $D \mathcal{F}(x)=D \Phi\left(x, F_{T}(x)\right)\left(I, Z_{T}\right)$. Early references are Goodman and Lance [1956], Morrison et al. [1962], and for applications to optimal control problems, Stoer and Bulirsch [1993].

As an example, consider the problem of computing periodic trajectories with a prescribed period $T$. This is a 
particular case of the above discussion where the mapping $F_{T}$ is in this context called the Poincaré map. Then $\mathcal{F}(x):=F_{T}(x)-x, D \mathcal{F}(x):=Z_{T}-I$, and so a zero $x$ is regular iff $Z_{T}$ has no eigenvalue equal to 1 .

\subsection{Switching times}

We will need to consider more general situations involving times at which both the state and dynamics may have jumps. It suffices to discuss the case of a single jump. We denote by e.g. $[y(t)]$ the jump of $y$ at time $t$. Given $f^{1}$ and $f^{2}: \mathbb{R}^{n} \rightarrow \mathbb{R}^{n}$, and $\varphi: \mathbb{R}^{n} \rightarrow \mathbb{R}^{n}$, all of them smooth, consider the following dynamical system

$$
\left\{\begin{aligned}
\dot{y}_{t} & =f^{1}\left(y_{t}\right), t \in(0, \tau) \\
\dot{y}_{t} & =f^{2}\left(y_{t}\right), t \in(\tau, T) \\
{[y(\tau)] } & =\varphi\left(y_{\tau_{-}}\right)
\end{aligned}\right.
$$

where the switching time $\tau \in(0, T)$ is for the moment a free variable. We compute the sensitivity of $y_{T}$ w.r.t. the initial condition $x$ and jump time $\tau$ as follows. We set $[f(\tau)]:=f^{2}\left(y_{\tau_{+}}\right)-f^{1}\left(y_{\tau_{-}}\right)$. Let $(\delta x, \delta \tau)$ be the perturbation of $(x, \tau)$. A key result is that the change in the value of $y$ at time $\tau+|\delta \tau|$, up to $o(|\delta x|+|\delta \tau|)$, is

$$
\varphi^{\prime}\left(y_{\tau_{-}}\right) z_{\tau_{-}}+\delta \tau\left(\varphi^{\prime}\left(y_{\tau_{-}}\right) f^{1}\left(y_{\tau_{-}}\right)-[f(\tau)]\right) .
$$

The first term does not depend on the variation of the switching time. The coefficient of $\delta \tau$ has two terms: the first takes into account the change in the jump, and the second the change in the dynamics. It easily follows that: Lemma 1. The directional derivative of $y_{T}$ w.r.t. $\left(y_{0}, \tau\right)$, in the direction $\left(\delta y_{0}, \delta \tau\right)$, is equal to $z_{T}$, where $z$ is Lischitz on $(0, \tau)$ and $(\tau, T)$, with jump at time $\tau$ denoted by $[z(\tau)]:=z_{\tau_{+}}-z_{\tau_{-}}$, and is solution of

$$
\left\{\begin{aligned}
\dot{z}_{t}= & D f^{1}\left(y_{t}\right) z_{t}, t \in(0, \tau) \\
\dot{z}_{t}= & D f^{2}\left(y_{t}\right) z_{t}, t \in(\tau, T) \\
z_{0}= & \delta y_{0} \\
{[z(\tau)]=} & \varphi^{\prime}\left(y_{\tau_{-}}\right) z_{\tau_{-}} \\
& +\delta \tau\left(\varphi^{\prime}\left(y_{\tau_{-}}\right) f^{1}\left(y_{\tau_{-}}\right)-[f(\tau)]\right) .
\end{aligned}\right.
$$

Let $g$ be a smooth mapping $\mathbb{R}^{n} \rightarrow \mathbb{R}$. It may happen that $\tau$ is defined as the first time for which the event $g\left(y_{\tau}\right)=0$ occurs. Denote by $F^{i}$ the flow associated with vector fields $f^{i}, i=1,2$. Applying the implicit function theorem (IFT) to the relation $g\left(F_{\tau}^{1}\left(y_{0}\right)\right)=0$, and defining $z^{1}$ as $z$ but with $f^{1}$ instead of $f$, we deduce that

Lemma 2. If $g^{\prime}\left(y_{\tau_{-}}\right) f^{1}\left(y_{\tau_{-}}\right) \neq 0$, then locally $\tau$ is a smooth function of $y_{0}$ with derivative $D \tau$ solution of

$$
g^{\prime}\left(y_{\tau}\right)\left(f^{1}\left(y_{\tau_{-}}\right) D \tau+z_{\tau}^{1}\right)=0 .
$$

We will call the condition $g^{\prime}\left(y_{\tau_{-}}\right) f_{1}\left(y_{\tau_{-}}\right) \neq 0$ a transversality condition, since it means that $y_{t}$ enters the manifold $g^{-1}(0)$ in a transversal way.

\section{UNCONSTRAINED OPTIMAL CONTROL}

\subsection{Framework}

We define the control and state spaces

$$
\mathcal{U}:=L^{\infty}\left(0, T, \mathbb{R}^{m}\right) ; \mathcal{Y}:=W^{1, \infty}\left(0, T, \mathbb{R}^{n}\right),
$$

where by $W^{1, \infty}\left(0, T, \mathbb{R}^{m}\right)$ we denote the set of primitives of functions in $L^{\infty}\left(0, T, \mathbb{R}^{n}\right)$. Let $f$ be $C^{\infty}$ and Lipschitz $\mathbb{R}^{m} \times \mathbb{R}^{n} \rightarrow \mathbb{R}^{n}$. The state equation is

$$
\dot{y}_{t}=f\left(u_{t}, y_{t}\right), t \in(0, T) ; y_{0}=y^{0},
$$

where the initial condition $y^{0}$ is given. For any $u \in \mathcal{U}$, it has a unique solution in $\mathcal{Y}$ denoted by $y[u]$. If $(u, y) \in \mathcal{U} \times \mathcal{Y}$ and $y=y[u]$, we say that $(u, y)$ is a trajectory. Consider the problem

$$
\operatorname{Min} \phi\left(y_{T}\right) ; \text { s.t. (5). }
$$

The Hamiltonian of the problem is $H[p](u, y):=p f(u, y)$, where $p \in \mathbb{R}^{n *}$ (dual of $\mathbb{R}^{n}$, whose elements are row vectors). The costate associated with the trajectory $(\bar{u}, \bar{y})$ is the solution of the (backward) costate equation

$$
-\dot{\bar{p}}_{t}=H\left[\bar{p}_{t}\right]\left(\bar{u}_{t}, \bar{y}_{t}\right), t \in(0, T) ; \bar{p}_{T}=D \phi\left(\bar{y}_{T}\right) .
$$

The Pontryagin's principle states that, if the trajectory $(u, y)$ is solution of $(P)$, with associated costate $p$, then $(u, y, p)$ is a Pontryagin extremal, i.e., for a.a. $t \in(0, T)$, the following Pontryagin inequality holds:

$$
H\left[p_{t}\right]\left(u_{t}, y_{t}\right) \leq H\left[p_{t}\right]\left(v, y_{t}\right), \text { for all } v \in \mathbb{R}^{m} .
$$

Let $(\bar{u}, \bar{y}, \bar{p})$ be a Pontryagin extremal with $\bar{u}$ continuous, and its associated costate. Then by (7):

$$
H_{u}\left[\bar{p}_{t}\right]\left(\bar{u}_{t}, \bar{y}_{t}\right)=0, \quad t \in[0, T],
$$

and also $D_{u u}^{2} H\left[\bar{p}_{t}\right]\left(\bar{u}_{t}, \bar{y}_{t}\right) \succeq 0$ for all $t \in[0, T]$ (where $\succeq$ stands for the Löwner order relation for symmetric matrices: $A \succeq 0$ iff $A$ is positive semidefinite). Assume that the strong Legendre condition holds, i.e., for a certain $\gamma>0$, and a.a. $t \in(0, T)$ :

$$
D_{u u}^{2} H\left[\bar{p}_{t}\right]\left(\bar{u}_{t}, \bar{y}_{t}\right) \succeq \gamma I .
$$

We deduce then from the IFT applied to the relation

$$
H_{u}\left[p_{t}\right](u, y)=0
$$

the existence of a smooth mapping $\Upsilon$ defined over an $L^{\infty}$ neigborhood $E$ of $\left\{\left(\bar{y}_{t}, \bar{p}_{t}\right), t \in[0, T]\right\}$ with image in $\mathbb{R}^{m}$, such that (10) holds in $E$ with $u$ close to $\bar{u}_{t}$ iff $u=\Upsilon(y, p)$ (the IFT provides an implicit function that is also local in time; however, we may add the time as a state variable, and then stick together the implicit functions for different times). Then $(\bar{y}, \bar{p})$ is solution for a.a. $t \in(0, T)$ of

$$
\left\{\begin{aligned}
\dot{y}_{t} & =f\left(\Upsilon\left(y_{t}, p_{t}\right), y_{t}\right), \\
-\dot{p}_{t} & =p_{t} f_{y}\left(\Upsilon\left(y_{t}, p_{t}\right), y_{t}\right),
\end{aligned}\right.
$$

with initial and final conditions

$$
y_{0}=y^{0} ; \quad p_{T}=D \phi\left(y_{T}\right) \text {. }
$$

We may then apply the shooting approach of the previous section. Since $y_{0}=y^{0}$ is given, we will define the shooting function as $\mathcal{S}\left(p_{0}\right):=D \phi\left(y_{T}\left[p_{0}\right]\right)-p_{T}\left[p_{0}\right]$, where by $\left(y_{t}\left[p_{0}\right], p_{t}\left[p_{0}\right]\right)$, we denote the solution of (11) at time $t$, with initial condition $\left(y^{0}, p_{0}\right)$. As we next show, that $\bar{p}_{0}$ is a regular zero of the shooting function appears to be intimately linked to the second order optimality conditions.

\subsection{Second order optimality conditions}

The linearized state equation is

$$
\dot{z}_{t}=D f\left(\bar{u}_{t}, \bar{y}_{t}\right)\left(v_{t}, z_{t}\right), \quad t \in(0, T) ; \quad z_{0}=0 .
$$

Given $v$ in $\mathcal{U}_{2}:=L^{2}\left(0, T, \mathbb{R}^{m}\right)$ (with norm denoted by $\left.\|\cdot\|_{2}\right)$, it has a unique solution in $\mathcal{Y}_{2}:=H^{1}\left(0, T, \mathbb{R}^{m}\right)$, denoted by $z[v]$. Set

$$
H(t):=H\left[\bar{p}_{t}\right]\left(\bar{u}_{t}, \bar{y}_{t}\right) ; H^{\prime}(t):=D H\left[\bar{p}_{t}\right]\left(\bar{u}_{t}, \bar{y}_{t}\right),
$$

etc. The quadratic form corresponding to the Hessian of Lagrangian of problem $(P)$ is denoted as follows, where $z=z[v]$ :

$$
Q_{0}(v):=\frac{1}{2} \int_{0}^{T} H^{\prime \prime}(t)\left(v_{t}, z_{t}\right)^{2} \mathrm{~d} t+\frac{1}{2} \phi^{\prime \prime}\left(\bar{y}_{t}\right)\left(z_{T}\right)^{2} .
$$


The following second order sufficient condition (SOSC) for local optimality

For some $\alpha^{\prime}>0, Q_{0}(v) \geq \alpha^{\prime}\|v\|_{2}^{2}$, for all $v \in \mathcal{U}_{2}$,

is known to imply the strong Legendre condition (9). Note that a second order necessary optimality condition $(S O N C)$ is that

$$
Q_{0}(v) \geq 0, \text { for all } v \in \mathcal{U}_{2} .
$$

We say that the quadratic growth condition holds whenever

$$
\left\{\begin{array}{l}
\text { For some } \alpha^{\prime \prime}>0, \\
\phi\left(\bar{y}_{T}\right)+\alpha^{\prime \prime}\|u-\bar{u}\|_{2}^{2} \leq \phi\left(y_{T}[u]\right) \\
\text { if }\|u-\bar{u}\|_{\infty} \text { is small enough. }
\end{array}\right.
$$

The following result is well known.

Theorem 3. Let $\bar{u}$ be a solution of $(P)$ satisfying the strong Legendre condition (9). Then the three following conditions are equivalent: (i) $\bar{p}_{0}$ is a regular zero of the shooting mapping, (ii) the second order condition (16), (iii) the quadratic growth condition (18)

In the sequel we will denote $\bar{f}(t):=f\left(\bar{u}_{t}, \bar{y}_{t}\right)$ as well as

$$
D \bar{f}(t):=D f\left(\bar{u}_{t}, \bar{y}_{t}\right) ; \bar{f}_{y}(t):=f_{y}\left(\bar{u}_{t}, \bar{y}_{t}\right),
$$

and similarly for other derivatives.

Proof We just give a sketch of the equivalence between (i) and (ii). Write (11) in the form, for $t \in(0, T)$ :

$$
\left\{\begin{aligned}
\dot{y}_{t} & =f\left(u_{t}, y_{t}\right), \\
-\dot{p}_{t} & =p_{t} f_{y}\left(u_{t}, y_{t}\right), \\
0 & =p_{t} f_{u}\left(u_{t}, y_{t}\right) .
\end{aligned}\right.
$$

Denote by $(v, z, q)$ the linearization of $(u, y, p)$ at the point $(\bar{u}, \bar{y}, \bar{p})$. Then the linearization of $(20)$ and (12) can be written in the form, for $t \in(0, T)$ (having in mind that the equalities in the second and third row are between row vectors):

$$
\left\{\begin{aligned}
\dot{z}_{t} & =D \bar{f}(t)\left(v_{t}, z_{t}\right), \\
-\dot{q}_{t} & =q_{t} \bar{f}_{y}(t)+v_{t}^{\top} H_{u y}(t)+z_{t}^{\top} H_{y y}(t), \\
0 & =q_{t} \bar{f}_{u}(t)+v_{t}^{\top} H_{u u}(t)+z_{t}^{\top} H_{y u}(t), \\
z_{0} & =0 ; \quad q_{T}=D^{2} \phi\left(y_{T}\right) z_{T} .
\end{aligned}\right.
$$

We recognize the optimality conditions for the problem of minimizing $Q_{0}(v)$. Since, by the $\operatorname{SOCN}(17), Q_{0}$ is a convex function, $\bar{p}_{0}$ is a regular zero of the shooting function iff 0 is the only critical point (i.e., point at which the derivative vanishes) of $Q_{0}$. Under (16), $Q_{0}$ is strictly convex, and hence, this holds.

On this subject, see Maurer and Pesch [1994] and its references who also present (i) the link to the sensitivity analysis (variation of the solution when the data of the problem are perturbed), and (ii) the link with the Riccati equation that allows to check numerically the sufficient second order conditions.

\subsection{Direct discretization and shooting}

The shooting approach is a convenient framework for analyzing the discretization of optimal control problems. We illustrate this in the case of the Euler method: the discretized problem is to minimize the final cost function $\phi\left(y_{N}\right)$, s.t. the discretized state equation

$$
y_{k+1}=y_{k}+h f\left(u_{k}, y_{k}\right), k=0, \ldots, N-1,
$$

where $h=T / N$. Writing the first order optimality conditions, we obtain a discrete costate equation and the stationarity of the Hamiltonian w.r.t. the control:

$$
\left\{\begin{aligned}
p_{k}-p_{k+1}= & h p_{k+1} f_{y}\left(u_{k}, y_{k}\right), \\
0= & p_{k+1} f_{u}\left(u_{k}, y_{k}\right), \\
& k=0, \ldots, N-1, \\
p_{N}= & \phi^{\prime}\left(y_{N}\right) .
\end{aligned}\right.
$$

We recognize in the r.h.s. the partial derivatives of the Hamiltonian w.r.t. the state and control. The second "algebraic" condition allows to eliminate the control in the same way as for the original problem, and so, we may express these conditions in the compact form

$$
\left\{\begin{array}{l}
y_{k+1}=y_{k}+h f\left(\Upsilon\left(y_{k}, p_{k+1}\right), y_{k}\right), \\
p_{k+1}=p_{k}-h p_{k+1} f_{y}\left(\Upsilon\left(y_{k}, p_{k+1}\right), y_{k}\right),
\end{array}\right.
$$

with end conditions

$$
y_{0}=y^{0} ; \quad p_{T}=D \phi\left(y_{N}\right) .
$$

The associated discrete shooting function is $\mathcal{S}_{N}\left(p_{0}\right):=$ $D \phi\left(y_{N}^{N}\left[p_{0}\right]\right)-p_{N}^{N}\left[p_{0}\right]$, where here by $\left(y^{N}\left[p_{0}\right], p^{N}\left[p_{0}\right]\right)$, we denote the solution of $(24)$ with initial condition $\left(y^{0}, p_{0}\right)$. By standard results on one-step integration solvers, $\mathcal{S}_{N}$ is well-defined for $N$ large enough if $p_{0}$ is close enough to $\bar{p}_{0}$, locally uniformly converges to $\mathcal{S}$, and the same holds for its Jacobian. In particular, $\mathcal{S}_{N}\left(\bar{p}_{0}\right) \rightarrow \mathcal{S}\left(\bar{p}_{0}\right)=0$, and we can show that for large enough $N$, Newton's method initialized at $\bar{p}_{0}$ converges to a locally unique zero $\bar{p}_{0}^{N}$ of $\mathcal{S}_{N}$, and since $\left|\mathcal{S}_{N}\left(\bar{p}_{0}\right)\right|=O(h)$, we deduce that $\mid \bar{p}_{0}^{N}-$ $\bar{p}_{0} \mid=O(h)$. It follows that the optimal control, state and costate of the discretized problem are at uniform (over time) $O(h)$ distance to the solution of the original problem. The result can be extended to Runge-Kutta methods of arbitrary order. However, the error order of the statecostate dynamics may be less than the order of the original Runge-Kutta method, as was established in Hager [2000], and generalized in Bonnans and Laurent-Varin [2006].

\section{CONTROL CONSTRAINED PROBLEMS}

\subsection{Framework}

Given a closed subset $U$ of $\mathbb{R}^{m}$, if we have a constraint of the form

$$
u_{t} \in U, \quad t \in(0, T),
$$

then the costate equation remains the same, but a Pontryagin extremal is now defined by, for a.a. $t \in(0, T)$ :

$$
H\left[p_{t}\right]\left(u_{t}, y_{t}\right) \leq H\left[p_{t}\right]\left(v, y_{t}\right) \text {, for all } v \in U \text {. }
$$

Let $(\bar{u}, \bar{y})$ be an optimal trajectory, with $\bar{u}$ continuous. Then for the associated costate $\bar{p}$, the Hamiltonian inequality (27) holds. Under some second order conditions, that are satisfied for strongly convex problems, this is again equivalent, in the vicinity of the trajectory $(\bar{u}, \bar{y})$, to a parametrization of the control of the form $u=\Upsilon(y, p)$, where $\Upsilon$ is a Lipschitz, and in general not differentiable, mapping. Then $(\bar{y}, \bar{p})$ is solution of, for a.a. $t \in(0, T)$ :

$$
\left\{\begin{aligned}
\dot{y}_{t} & =f\left(\Upsilon\left(y_{t}, p_{t}\right), y_{t}\right) \\
-\dot{p}_{t} & =p_{t} f_{y}\left(\Upsilon\left(y_{t}, p_{t}\right), y_{t}\right) .
\end{aligned}\right.
$$

So, we can define the shooting function

$$
\mathcal{S}\left(p_{0}\right):=\phi^{\prime}\left(y_{T}\left[p_{0}\right]\right)-p_{T}\left[p_{0}\right],
$$

where $\left(y_{T}\left[p_{0}\right], p_{T}\left[p_{0}\right]\right)$ is the final value of the solution of the above system with initial solution $\left(y^{0}, p_{0}\right)$. The shooting function is locally Lipschitz and in general nonsmooth. 
Some results of differential stability can nevertheless be derived, see Malanowski [1984], but using an infinite dimensional framework.

\subsection{Parameterization of switching times}

For simplicity we discuss the case of a single control (i.e., $m=1)$ subject to the constraint

$$
u_{t} \geq 0, \quad t \in(0, T),
$$

in the case when an optimal trajectory $(\bar{u}, \bar{y})$, with $\bar{u}$ continuous, is such that a constrained arc is followed by an unconstrained one, the junction time being $\bar{\tau} \in(0, T)$. Given a trajectory $(u, y)$ close to $(\bar{u}, \bar{y})$ and $\tau$ close to $\bar{\tau}$, we denote by $\Upsilon^{U}(y, p)$ the control that attains the unconstrained minimum of the Hamiltonian (assumed to be welldefined). The junction time $\tau$ satisfies $\Upsilon^{U}\left(\bar{y}_{\tau}, \bar{p}_{\tau}\right)=0$, or (locally) equivalently, $\bar{p}_{\tau} f_{u}\left(0, \bar{y}_{\tau}\right)=0$. Setting $\Xi(u, y, p):=$ $\left(f(u, y),-p f_{y}(u, y)\right)$, we see that the optimality system implies

$$
\left\{\begin{array}{c}
\left(\dot{\bar{y}}_{t}, \dot{\bar{p}}_{t}\right)=\Xi\left(0, \bar{y}_{t}, \bar{p}_{t}\right), \quad t \in(0, \tau), \\
\left(\dot{\bar{y}}_{t}, \dot{\bar{p}}_{t}\right)=\Xi\left(\Upsilon^{U}\left(\bar{y}_{t}, \bar{p}_{t}\right), \bar{y}_{t}, \bar{p}_{t}\right), t \in(\tau, T), \\
\bar{y}_{0}=y^{0} ; \bar{p}_{T}=D \phi\left(\bar{y}_{T}\right) ; \bar{p}_{\tau} f_{u}\left(0, \bar{y}_{\tau}\right)=0,
\end{array}\right.
$$

as well as the complementarity conditions

$$
\left\{\begin{aligned}
\bar{p}_{t} f_{u}\left(\bar{u}_{t}, \bar{y}_{t}\right) \geq 0 & \text { over }(0, \tau), \\
\bar{u}_{t} \geq 0 & \text { over }(\tau, T) .
\end{aligned}\right.
$$

We will assume the "strict" complementarity conditions

$$
\left\{\begin{aligned}
\bar{p}_{t} f_{u}\left(\bar{u}_{t}, \bar{y}_{t}\right)>0 & \text { over }[0, \tau), \\
\bar{u}_{t}>0 & \text { over }(\tau, T],
\end{aligned}\right.
$$

as well as the transversality condition

$$
\frac{\mathrm{d}}{\mathrm{d} t} \Upsilon^{U}\left(y_{\tau}, p_{\tau}\right)>0
$$

One can show that the analysis of section 2.2 applies: $\Upsilon^{U}\left(y_{\tau}, p_{\tau}\right)=0$ is locally equivalent to $\tau=\tau^{U}\left(y_{\tau}, p_{\tau}\right)$, for some smooth function $\tau^{U}$. It follows that $\tau$ is a smooth function of $p_{0}$, and hence, the shooting mapping is (locally) well-defined and smooth.

\subsection{Regularity of the solution of the shooting function}

Define the critical cone by

$$
C(\bar{u}):=\left\{v \in \mathcal{U}_{2} ; v=0 \text { on }(0, \tau)\right\} .
$$

The the following SONC hold:

$$
Q_{0}(v) \geq 0, \text { for all } v \in C(\bar{u}) \text {. }
$$

Consider the following second order (sufficient for local optimality) condition:

For some $\alpha^{\prime}>0, Q_{0}(v) \geq \alpha^{\prime}\|v\|^{2}$, for all $v \in C(\bar{u})$.

Since $\bar{u}$ is continuous, it implies the strong Legendre condition similar to (9) but over $[\tau-\varepsilon, T]$, for some $\varepsilon>0$ : there exists $\gamma>0$ such that

$$
D_{u u}^{2} H\left[\bar{p}_{t}\right]\left(\bar{u}_{t}, \bar{y}_{t}\right) \succeq \gamma I, \quad \text { for all } t \in[\tau-\varepsilon, T] .
$$

Theorem 4. Let (34) and (37) hold, with $\bar{u}$ continuous. Then $\bar{p}_{0}$ is a regular solution of the shooting function.

Proof Consider the linearized shooting equations, obtained by applying lemma 1 . Since $\bar{u}$ is continuous, there is by (3) no jump in the linearized state and costate, which therefore are still solution of the last and two first rows of $(21)$ over $(0, T)$, while the third one holds on $(\tau, T)$.
Similarly to the case of an unconstrained control, this can be interpreted as the optimality condition of minimizing $Q_{0}(\cdot)$ over the critical cone defined in (35). By (37), this holds only when $v=0$, implying that $(z, q)=0$. Linearizing the condition $\Upsilon^{U}\left(y_{\tau}, p_{\tau}\right)=0$, since $(v, z, q)=0$ we obtain with (34) that the linearization of $\tau$ equals 0 . The conclusion follows.

This analysis is relatively easily extended to the case of several mixed state and control constraints, see Maurer and Pesch [1995], Malanowski et al. [1998]. The analysis is more involved when jumps occur in the state-costate dynamics, as in the case when the control is not continuous, see Milyutin and Osmolovskiu [1998]. Jumps can also occur in the costate dynamics when state constraints are present, as discussed in section 5 .

\subsection{Direct discretization and shooting}

We continue with the simple situation of the previous section. If (33)-(34) and (38) hold, it can be proved that the Euler discretization of the problem has a unique solution $u^{h}$ uniformly close to $\bar{u}$, with again the control constraint active after a certain discrete time converging to $\bar{\tau}$. The construction of the solution can be based on an homotopy method. Setting $t_{k}=k h, k=0$ to $N$, and

$$
\left(\hat{u}_{k}, \hat{y}_{k}, \hat{p}_{k+1}\right)=\left(\bar{u}_{t_{k}}, \bar{y}_{t_{k}}, \bar{p}_{t_{k}}\right), \quad k=0, \ldots, N-1,
$$
we obtain an approximate solution of the discrete optimality system. We define a path parameterized by $\theta \in[0,1]$ as follows. Consider the perturbed state equation

$$
y_{k+1}=y_{k}+h f\left(u_{k}, y_{k}\right)+\theta h \delta_{k}^{P}, k=0, \ldots, N-1,
$$

and an "optimality system" defined by

$$
\left\{\begin{aligned}
& p_{k}-p_{k+1}= h p_{k+1} f_{y}\left(u_{k}, y_{k}\right)+\theta h \delta_{k}^{D} \\
& 0 \leq p_{k+1} f_{u}\left(u_{k}, y_{k}\right), u_{k} \geq 0 \\
& 0=p_{k+1} f_{u}\left(u_{k}, y_{k}\right) u_{k} \\
& k=0, \ldots, N-1 \\
& p_{N}=\phi^{\prime}\left(y_{N}\right) \theta+\delta_{N}^{D} ; y_{0}=y^{0}
\end{aligned}\right.
$$

We fix $\delta^{P}$ and $\delta^{D}$ (where " $P$ " and " $D$ " stand for primal and dual) in such a way that these equations are satisfied by $\left(\hat{u}_{k}, \hat{y}_{k}, \hat{p}_{k+1}\right)$ when $\theta=1$ (note that the condition of stationarity of the Hamiltonian w.r.t. the control needs no perturbation term). By standard Taylor expansions, we get that $\left\|\left(\delta^{P}, \delta^{D}\right)\right\|_{\infty}=O(h)$. We now look for a path of solutions $\left(u^{\theta}, y^{\theta}, p^{\theta}\right)$ solution of (40)-(41), which will provide a solution of the discrete optimality system for $\theta=0$.

Theorem 5. Under the previous assumptions, there exists a path $\left(u^{\theta}, y^{\theta}, p^{\theta}\right)$ solution of $(40)-(41)$, for $\theta \in[0,1]$, at $L^{\infty}$ distance of $(\hat{u}, \hat{y}, \hat{p})$ of order $h$.

Proof Assume that the path is well-defined and at $L^{\infty}$ distance of $(\bar{u}, \bar{y}, \bar{p})$ of order $h^{1 / 2}$ (to begin with) at least up to some $\theta \in(0,1]$. By the second order optimality conditions, $\left(u^{\theta}, y^{\theta}, p^{\theta}\right)$ is a local solution of the perturbed discretized problem defined by the state equation (40), the control constraints $u_{k} \geq 0, k=0$ to $N-1$, and the cost function (perturbed by a linear term)

$$
\phi\left(y_{N}\right)+\theta\left(\sum_{k=0}^{N-1} \delta_{k}^{D} y_{k}+\delta_{N}^{D} y_{N}\right)
$$

By Jittorntrum [1984] or [Bonnans and Shapiro, 2000, Thm 5.53], $u^{\theta}$ has a directional derivative say $v$ w.r.t. $\theta$; it 
suffices to prove that the latter has an $L^{\infty}$ norm of order $h$, since then (by the discrete Gronwall lemma) the same order is obtained for the directional derivatives $(z, q)$ of the state and costate, and this will prove that the Lipschitz constant of $\theta \mapsto\left(u^{\theta}, y^{\theta}, p^{\theta}\right)$ (in the $L^{\infty}$ norm) is of order $h$; the conclusion will follow.

Since the direction minimizes a quadratic cost (which is nothing but the Hessian of Lagrangian of the discrete problem), by comparing it first to the zero direction (which is feasible since the control constraints are not perturbed), and using (37), we can prove that $\|v\|_{2}=O(h)$, where $\|v\|_{2}^{2}:=h \sum_{k} v_{k}^{2}$. The $L^{\infty}$ estimates for $(z, q)$ easily follow. Finally, by the linearization of the stationarity of the Hamiltonian w.r.t. the control, we can express $v_{k}$ as a smooth function of $\left(z_{k}, q_{k+1}\right)$; the $L^{\infty}$ estimates for $v$ follows.

Remark 6. The previous arguments seem to be based on the homotopy idea more than the shooting itself. Still, one can check that under the previous assumptions, the shooting mapping for the discretized problem is welldefined, but it will not be differentiable in general. On the other hand, the previous discussion gives a way to compute its directional derivatives and so we may as well speak of an homotopy on the shooting mapping.

\section{STATE CONSTRAINED PROBLEMS}

\subsection{Framework}

We restrict the analysis to the case of a single state constraint

$$
\left\{\begin{array}{l}
\operatorname{Min} \phi\left(y_{T}\right) ; \quad \text { s.t. } \\
\dot{y}_{t}=f\left(u_{t}, y_{t}\right), \quad t \in[0, T] \\
g\left(y_{t}\right) \leq 0, \quad t \in[0, T] ; \quad y_{0}=y^{0},
\end{array}\right.
$$

with $g: \mathbb{R}^{n} \rightarrow \mathbb{R}$ smooth, and $g\left(y^{0}\right)<0$. Let $\bar{u}$ be a continuous solution with associated state $\bar{y}$ and contact set

$$
I(\bar{u}):=\left\{t \in[0, T] ; g\left(\bar{y}_{t}\right)=0\right\} .
$$

We say that $\mu \in M([0, T]$ ) (space of Borel measures over $[0, T])$ is complementary to the state constraint if we have

$$
\mathrm{d} \mu \geq 0 ; \quad g(\bar{y}) \leq 0 ; \quad \int_{0}^{T} g(\bar{y}) \mathrm{d} \mu_{t}=0 .
$$

With $\mu \in M([0, T])$ we associated the costate $p \in$ $B V\left(0, T, \mathbb{R}^{n *}\right.$ ) (where $B V(0, T)$ is the space of functions with bounded variation over $[0, T])$, uniquely defined by

$$
\left\{\begin{aligned}
-\mathrm{d} p_{t} & =p_{t} D_{y} \bar{f}(t) \mathrm{d} t+g^{\prime}\left(\bar{y}_{t}\right) \mathrm{d} \mu_{t}, \\
p_{T} & =D \phi\left(\bar{y}_{T}\right) .
\end{aligned}\right.
$$

We say that $\mu$ is a Pontryagin multiplier if the associated costate $p$ is such that the Pontryagin inequality (7) holds. Lemma \%. If the following qualification condition holds:

$$
g^{\prime}(\bar{y}) z[\hat{v}]<0 \text { over } I(\bar{u}) \text {, for some } \hat{v} \in \mathcal{U}
$$

then the set of Pontryagin multipliers is nonempty and bounded.

Proof See e.g. Bonnans and Hermant [2007].

On a constrained arc, we have two algebraic equations: $D_{u} H\left[p_{t}\right]\left(\bar{u}_{t}, \bar{y}_{t}\right)=0$ and either $g\left(\bar{y}_{t}\right)=0$, or its differentiated form $g^{\prime}\left(\bar{y}_{t}\right) f\left(\bar{u}_{t}, \bar{y}_{t}\right)=0$. However, both equations determine $\bar{u}_{t}$, but not the density $\lambda_{t}$ of the measure $\mathrm{d} \mu$.
This leads to the so-called alternative formulation of the optimality system, that takes its origin in the Soviet school [Pontryagin et al., 1986, Ch. VI], was first introduced in Bryson et al. [1963], improved in Jacobson et al. [1971], given a mathematically rigorous setting in Maurer [1979], and generalized in Bonnans and Hermant [2009].

\subsection{The alternative optimality system I}

Set $\eta^{1}:=-\mu$ and define the alternative costate $p^{1}:=p-$ $\eta^{1} g^{\prime}(\bar{y})$. The "total derivative" of the state constraint is defined as $g^{(1)}(u, y):=g^{\prime}(y) f(u, y)$. One easily shows, using the identity

$$
g_{y}^{(1)}(u, y)=g^{\prime \prime}(y) f(u, y)+g^{\prime}(y) f_{y}(u, y)
$$

that $p^{1}$ has a bounded derivative given by

$$
\left\{\begin{aligned}
-\dot{p}_{t}^{1} & =p \bar{f}_{y}(t)+\eta^{1} g^{\prime \prime}\left(\bar{y}_{t}\right) f\left(\bar{u}_{t}, \bar{y}_{t}\right), \\
& =p^{1} \bar{f}_{y}(t)+\eta^{1} g_{y}^{(1)}\left(\bar{u}_{t}, \bar{y}_{t}\right) .
\end{aligned}\right.
$$

Define the alternative Hamiltonian as

$$
H^{1}\left[p^{1}, \eta^{1}\right](u, y):=p^{1} f(u, y)+\eta^{1} g^{(1)}(u, y) .
$$

Then the dynamics of the alternative costate has the form

$$
-\dot{p}_{t}^{1}=H_{y}^{1}\left[p_{t}^{1}, \eta_{t}^{1}\right]\left(u_{t}, y_{t}\right)
$$

On the other hand, along the nominal trajectory $(\bar{u}, \bar{y})$ we have that for all $t \in(0, T)$ and $v \in \mathbb{R}^{m}$

$$
H^{1}\left[p_{t}^{1}, \eta_{t}^{1}\right]\left(v, \bar{y}_{t}\right)=\bar{p}_{t} f\left(v, \bar{y}_{t}\right),
$$

and so the original Pontryagin inequality (7) is equivalent to the alternative Pontryagin inequality for a.a. $t$ :

$$
H^{1}\left[p_{t}^{1}, \eta_{t}^{1}\right]\left(\bar{u}_{t}, \bar{y}_{t}\right) \leq H^{1}\left[p_{t}^{1}, \eta_{t}^{1}\right]\left(v, \bar{y}_{t}\right), \quad \forall v \in \mathbb{R}^{m} .
$$

The corresponding stationarity equation w.r.t. the control reads

$$
p_{t}^{1} f_{u}\left(\bar{u}_{t}, \bar{y}_{t}\right)+\eta_{t}^{1} g_{u}^{(1)}\left(\bar{u}_{t}, \bar{y}_{t}\right)=0
$$

For a first order state constraint, i.e., if $g_{u}^{(1)}\left(\bar{u}_{t}, \bar{y}_{t}\right) \neq 0$, for all $t \in[0, T]$, when the constraint is active, we can eliminate $\eta^{1}$ from this equation and get the control from the equation $g^{(1)}\left(\bar{u}_{t}, \bar{y}_{t}\right)=0$. So the two algebraic variables can be eliminated as function of the state and costate. Otherwise we need to push one step further the idea of alternative optimality system, as we will now show.

\subsection{The alternative optimality system II}

Assume that the state constraint is not of first order, more precisely, that $g_{u}^{(1)}(v, y)=0$, for all $(v, y) \in \mathbb{R}^{m} \times$ $\mathbb{R}^{n}$. We can then write $g^{(1)}$ as function of $y$ only, with total derivative $g^{(2)}(u, y):=g_{y}^{(1)}(y) f(u, y)$. Let $\eta^{2}$ be any primitive of $-\eta^{1}$. The second alternative costate and Hamiltonian are defined resp. as

$$
\left\{\begin{array}{l}
p_{t}^{2}:=p_{t}^{1}-\eta^{2} g_{y}^{(1)}\left(\bar{y}_{t}\right), \quad t \in[0, T] \\
H^{2}\left[p^{2}, \eta^{2}\right](u, y):=p^{2} f(u, y)+\eta^{2} g^{(2)}(u, y)
\end{array}\right.
$$

Using

$$
g_{y}^{(2)}(u, y)=g_{y y}^{(1)}(y) f(u, y)+g_{y}^{(1)}(y) f_{y}(u, y)
$$

and (51) as well as $-\dot{\eta}_{t}^{2}=\eta_{t}^{1}$, we get, skipping arguments:

$$
\left\{\begin{aligned}
-\dot{p}_{t}^{2} & =-\dot{p}_{t}^{1}-\eta_{t}^{1} g_{y}^{(1)}+\eta_{t}^{2} g_{y y}^{(1)} \bar{f} \\
& =p_{t}^{2} \bar{f}_{y}(t)+\eta_{t}^{2}\left(g_{y}^{(1)} f_{y}+g_{y y}^{(1)} \bar{f}\right) \\
& =H_{y}^{2}\left[p_{t}^{2}, \eta_{t}^{2}\right]\left(\bar{u}_{t}, \bar{y}_{t}\right) .
\end{aligned}\right.
$$


Again, the original Pontryagin inequality (7) is equivalent to the one for the alternative Hamiltonian, and the condition of stationarity w.r.t. the control reads

$$
p_{t}^{2} \bar{f}_{u}(t)+\eta_{t}^{2} g_{u}^{(2)}\left(\bar{u}_{t}, \bar{y}_{t}\right)=0 \text {. }
$$

For a second order state constraint, i.e., when

$$
g_{u}^{(2)}\left(\bar{u}_{t}, \bar{y}_{t}\right) \neq 0, \text { for all } t \in[0, T],
$$

we can recover $\eta_{t}^{2}$ from this equation, and the control from the equation $g^{(2)}\left(\bar{u}_{t}, \bar{y}_{t}\right)=0$. We also note that (59) implies the uniqueness of the multiplier $\mathrm{d} \mu$, and therefore also of the costate $p$. We will see how to use the alternative formulations in the design of a shooting algorithm. The case of a first-order state constraint is presented in Malanowski and Maurer [1998]. We detail next the case of a second order state constraint.

\subsection{Shooting for second-order state constraints}

We assume that (i) we have a second-order state constraint, not active at time 0 , and in that case the qualification condition (47) holds, and (ii) the uniform strong Legendre condition:

$$
\text { For some } \alpha^{\prime \prime}>0, H_{u u}\left[\bar{p}_{t}\right]\left(\bar{u}_{t}, \bar{y}_{t}\right) \succeq \alpha^{\prime \prime} I \text {. }
$$

We concentrate on the case when an optimal trajectory $(\bar{u}, \bar{y})$ is such that an unconstrained arc is followed by a constrained one, itself followed by an unconstrained one, with junction times denoted by $\left(\tau_{1}, \tau_{2}\right)$, and $0<\tau_{1}<\tau_{2}<$ $T$. We next apply the alternative formulation on each of the three arcs, with different integration constants for $\eta^{1}$ and $\eta^{2}$ on each arc. For unconstrained arcs (where the $\eta^{i}$ are constant) we choose $\eta^{1}=\eta^{2}=0$, so that $p=p^{2}$, and on the constrained arc, we choose $\eta^{1}$ and $\eta^{2}$ so that $p^{2}$ is continuous at the exit time $\tau_{2}$. Since

$$
p_{t}^{2}=p_{t}-\eta^{1} g^{\prime}\left(\bar{y}_{t}\right)-\eta^{2} g_{y}^{(1)}\left(\bar{y}_{t}\right)
$$

and $[p(t)]=-[\mu(t)] g^{\prime}\left(\bar{y}_{t}\right)$, the jumps of $p^{2}$ (skipping arguments) satisfy

$$
-\left[p^{2}\right]=\left([\mu]+\left[\eta^{1}\right]\right) g^{\prime}+\left[\eta^{2}\right] g_{y}^{(1)} .
$$

The state constraint being of second order, when it is active, we have that, see Bonnans and Hermant [2009]:

$$
g^{\prime}\left(\bar{y}_{t}\right) \text { and } g_{y}^{(1)}\left(\bar{y}_{t}\right) \text { are linearly independent. }
$$

So we have the unique decomposition at the entry and exit time of the constrained arc:

$$
-\left[p^{2}\left(\tau_{1}\right)\right]=\nu_{1} g^{\prime}+\nu_{2} g_{y}^{(1)} ;\left[p^{2}\left(\tau_{2}\right)\right]=0,
$$

with

$$
\left\{\begin{array}{l}
\nu_{1}=\left[\mu\left(\tau_{1}\right)\right]+\eta_{\tau_{1}+}^{1} ; \nu_{2}=\eta_{\tau_{1}+}^{2} \\
0=\left[\mu\left(\tau_{2}\right)\right]-\eta_{\tau_{2}-}^{1}=-\eta_{\tau_{2}-}^{2}
\end{array}\right.
$$

Note that $\eta^{2}$ is continuous at time $\tau_{2}$ (while in general $\eta^{1}$ is not). Then the algebraic relations on the unconstrained arcs are

$$
p_{t} \bar{f}_{u}(t)=0 ; \quad \eta_{t}^{2}=0,
$$

and on the constrained arc

$$
\left\{\begin{array}{l}
p_{t}^{2} \bar{f}_{u}(t)+\eta_{t}^{2} g_{u}^{(2)}\left(\bar{u}_{t}, \bar{y}_{t}\right)=0 \\
g^{(2)}\left(\bar{u}_{t}, \bar{y}_{t}\right)=0
\end{array}\right.
$$

The shooting mapping: $\mathbb{R}^{n+4} \rightarrow \mathbb{R}^{n+4}$ is as follows:

$$
\mathcal{S}:\left(\begin{array}{c}
p_{0} \\
\nu_{1} \\
\nu_{2} \\
\tau_{1} \\
\tau_{2}
\end{array}\right) \mapsto\left(\begin{array}{c}
\phi^{\prime}\left(y_{T}\right)-p_{T} \\
g\left(y_{\tau_{1}}\right) \\
g^{(1)}\left(y_{\tau_{1}}\right) \\
g^{(2)}\left(\Upsilon_{\tau_{1-}}, y_{\tau_{1}}\right) \\
g^{(2)}\left(\Upsilon_{\tau_{2+}}, y_{\tau_{2}}\right)
\end{array}\right),
$$

where by $\Upsilon_{t}:=\Upsilon\left(p_{t}^{2}, \eta_{t}^{2}, y_{t}\right)$ and $\Upsilon\left(p^{2}, \eta^{2}, y\right)$ is the function implicitly defined by (compare to (58))

$$
p^{2} f_{u}(\Upsilon, y)+\eta^{2} g_{u}^{(2)}(\Upsilon, y)=0
$$

\subsection{Analysis of the shooting mapping}

We only give an informal presentation of the main ideas of the result, due to Bonnans and Hermant [2007] and based on the analysis of the optimality conditions of the following auxiliary optimization problem, parameterized by the junction times:

$$
\left\{\begin{array}{l}
\operatorname{Min} \phi\left(y_{T}\right) \text { s.t. state equation }(5) \text { and } \\
g\left(y_{\tau_{1}}\right)=g^{(1)}\left(y_{\tau_{1}}\right)=0, \\
g^{(2)}(u, y)=0 \text { over }\left(\tau_{1}, \tau_{2}\right)
\end{array} \quad\left(P\left(\tau_{1}, \tau_{2}\right)\right)\right.
$$

It has a mixed constraint (i.e., a constraint that involves both the state and costate). Identifying $\left(-\nu_{1},-\nu_{2}\right)$ with the multipliers associated with the two state constraints at the entry point, and denoting by $p^{2}, \eta^{2}$ the costate and multiplier for the state constraint, and by $\mathcal{S}_{i: j}$ the restriction to components $i$ to $j$ of the shooting mapping, we recover (57), (64) and (66)-(67), and deduce that:

Lemma 8. The optimality system of $\left(P\left(\tau_{1}, \tau_{2}\right)\right)$ coincides with the zeros of $\mathcal{S}_{1: 3}$.

The next lemma, which is a key result, is not just a corollary of the previous one, due to the variation of junction times.

Lemma 9. The linearization of the optimality system of $\left(P\left(\tau_{1}, \tau_{2}\right)\right)$ coincides (in a sense made precise in the proof) with the linearization of the zeros of $\mathcal{S}_{1: 3}$.

Proof We detail the only delicate point, which is the linearization at time $\tau_{1}$ of the first relation in (64). By (64)-(65), we get, skipping arguments and setting $g=g^{0}$ :

$$
-\left[\dot{p}^{2}\right]=\left[p^{2}\right] \bar{f}_{y}+\left[\eta^{2}\right] g_{y}^{(2)}=-\sum_{i=1}^{2} \nu_{i} g_{y}^{(i-1)} \bar{f}_{y}+\nu_{2} g_{y}^{(2)} \text {. }
$$

With lemma 1, we deduce that:

$$
-\left[q^{2}\left(\tau_{1}\right)\right]=\sum_{i=1}^{2}\left(\delta \nu_{i} g_{y}^{(i-1)}+\nu_{i} z_{\tau_{1}}^{\top} g_{y y}^{(i-1)}\right)+\delta \tau_{1} X,
$$

where the coefficient of $\delta \tau$ is

$$
\left\{\begin{aligned}
X & =\left[\dot{p}^{2}\right]-\frac{\mathrm{d}}{\mathrm{d} t}\left[p^{2}\right], \\
& =\sum_{i=1}^{2} \nu_{i}\left(g_{y}^{(i-1)} \bar{f}_{y}+g_{y y}^{(i-1)} \bar{f}\right)-\nu^{2} g_{y}^{(2)}, \\
& =\sum_{i=1}^{2} \nu_{i} g_{y}^{(i)}-\nu_{2} g_{y}^{(2)}=\nu_{1} g_{y}^{(1)} .
\end{aligned}\right.
$$

We finally get

$$
\left\{\begin{array}{l}
-\left[q^{2}\left(\tau_{1}\right)\right]=\sum_{i=1}^{2}\left(\nu_{i} z_{\tau_{1}}^{\top} g_{y y}^{(i-1)}+\lambda^{i} g_{y}^{(i-1)}\right) \\
\lambda^{1}=\delta \nu_{1} ; \quad \lambda^{2}=\delta \nu_{2}+\delta \tau_{1} \nu_{1} .
\end{array}\right.
$$

We see that we recover the linearization of the optimality conditions of the auxiliary problem $\left(P\left(\tau_{1}, \tau_{2}\right)\right)$, the parameters $\left(\lambda^{1}, \lambda^{2}\right)$ being interpreted as the opposite of the linearization of the Lagrange multipliers associated with the two entry point conditions of $\left(P\left(\tau_{1}, \tau_{2}\right)\right)$. 
We can now perform a local analysis of the shooting function, assuming that

$$
\frac{\mathrm{d}}{\mathrm{d} t} g^{(2)}\left(\bar{u}_{t \pm}, \bar{y}_{t}\right) \neq 0, \quad t=\tau_{1-}, \tau_{2+},
$$

(which in the case of a scalar control, is equivalent to the discontinuity of the control at the junction times) and to the following second order optimality conditions. First we defined the quadratic form corresponding to the Hessian of Lagrangian of the problem, i.e.

$$
Q(v):=Q_{1}(v)+\int_{0}^{T} g^{\prime \prime}\left(\bar{y}_{t}\right)\left(z_{t}\right)^{2} \mathrm{~d} \mu_{t}
$$

Then we define the strict critical cone as

$$
C_{S}(\bar{u}):=\left\{v \in \mathcal{U}_{2} ; g^{\prime}\left(\bar{y}_{t}\right) z_{t}=0, t \in\left[\tau_{1}, \tau_{1}\right]\right\} .
$$

and assume that for some $\alpha>0$

$$
Q(v) \geq \alpha\|v\|_{2}^{2}, \text { for all } v \in C_{S}(\bar{u})
$$

Note that a second order necessary condition is that (77) holds with $\alpha=0$.

Theorem 10. If (74) and (77) hold, $\left(\bar{p}_{0}, \nu_{1}, \nu_{2}, \tau_{1}, \tau_{2}\right)$ is a regular zero of the shooting function.

Proof We have to prove that $D \mathcal{S}$ is injective at the solution. Let $\left(\delta p_{0}, \delta \nu_{1}, \delta \nu_{2}, \delta \tau_{1}, \delta \tau_{2}\right)$ belong to its kernel. First, using (73), we deduce from the previous lemma that $-\lambda$ correspond to the multipliers of the linearized optimality system of $\left(P\left(\tau_{1}, \tau_{2}\right)\right)$. One can show that the latter coincides with the optimality conditions for the minimization of $Q(\cdot)$ over $C_{S}(\bar{u})$. By $(77)$ it follows that $\left(\delta p_{0}, \delta \nu_{1}, \lambda\right)=0$ and the corresponding linearization $(v, z, q)$ of the control, state and costate is also equal to 0 . Then, using the expression of $D \mathcal{S}_{4: 5}$, and (74), we deduce from the linearization of conditions $\mathcal{S}_{4: 5}$, which since $(v, z)=0$ boils down to e.g. $\delta \tau_{1} \frac{\mathrm{d}}{\mathrm{d} t} g^{(2)}\left(\bar{u}_{\tau_{1}-}, \bar{y}_{\tau_{1}}\right)=0$, that $\left(\delta \tau_{1}, \delta \tau_{2}\right)=0$, which since $\lambda=0$ gives $\delta \nu=0$ in view of $(73)$.

\subsection{Touch points: the reduction approach}

An isolated point of the contact set defined in (44) is called a touch point. We next consider the case when the contact set reduces to a unique touch point $\tau \in(0, T)$. The idea of reduction is as follows. For $\varepsilon>0$ small enough, set $\tau_{1}:=\tau-\varepsilon, \tau_{2}:=\tau+\varepsilon$, and for $u \in \mathcal{U}$ and $t \in\left[\tau_{1}, \tau_{2}\right]$, define $G(u, t):=g\left(y_{t}[u]\right)$, and

$$
\Theta(u):=\max \left\{G(u, t), \quad t \in\left[\tau_{1}, \tau_{2}\right]\right\} .
$$

We assume the reducibility condition

$$
\gamma:=\ddot{g}\left(\bar{y}_{t}\right)_{t=\tau}<0 .
$$

From standard results on the perturbation theory of unconstrained maximization problems [Bonnans and Shapiro, 2000, Sections 4.3 and 5.2] we know that since $g\left(\bar{y}_{t}\right)$ attains its maximum at a unique time $\tau$ in $\left[\tau_{1}, \tau_{2}\right], \Theta$ has a Fréchet derivative defined by

$$
D \Theta(\bar{u}) v=G_{u}(\bar{u}, \tau) v=g^{\prime}\left(\bar{y}_{t}\right) z_{\tau}[v] .
$$

In addition, if the constraint is of order at least 3 , then we have the second derivative

$$
\Theta^{\prime \prime}(\bar{u})(v, v)=\max _{\delta \tau} G^{\prime \prime}(\bar{u}, \tau)(v, \delta \tau) .
$$

We have that

$$
\left\{\begin{aligned}
G^{\prime \prime}(\bar{u}, \tau)(v, \delta \tau)= & g^{\prime}\left(\bar{y}_{\tau}\right) z_{\tau}^{2}+g^{\prime \prime}\left(\bar{y}_{\tau}\right)\left(z_{\tau}\right)^{2} \\
& +2 \delta \tau g_{y}^{(1)}\left(\bar{y}_{\tau}\right) z_{\tau}+\gamma(\delta \tau)^{2}
\end{aligned}\right.
$$

where the second derivative $z^{2}$ of $\bar{y}[u]$ at $\bar{u}$ in the direction $v$ satisfies

$$
\dot{z}_{t}^{2}=\bar{f}_{y} z^{2}+\bar{f}^{\prime \prime}(v, z)^{2}, t \in(0, T) ; \quad z_{0}^{2}=0 .
$$

Maximizing w.r.t. $\delta \tau$, we obtain

$\Theta^{\prime \prime}(\bar{u})(v, v)=g^{\prime}\left(\bar{y}_{\tau}\right) z_{\tau}^{2}+g^{\prime \prime}\left(\bar{y}_{\tau}\right)\left(z_{\tau}\right)^{2}-\gamma^{-1}\left(g_{y}^{(1)}\left(\bar{y}_{\tau}\right) z_{\tau}\right)^{2}$.

It happens that, for a second order state constraint, $\Theta(\bar{u})$ still has a second order Taylor expansion with the above expression of $\Theta(\bar{u})(v, v)$, although is is no more $C^{2}$. Obviously, $\bar{u}$ is a local solution of the problem

$$
\operatorname{Min}_{u \in \mathcal{U}} \phi\left(y_{T}[u]\right) ; \quad \Theta(u) \leq 0 .
$$

The corresponding quadratic form (Hessian of Lagrangian) can be written as follows:

$$
Q_{2}(v):=Q_{1}(v)+\frac{1}{2} \beta\left(g^{\prime \prime}\left(\bar{y}_{\tau}\right)\left(z_{\tau}\right)^{2}-\gamma^{-1}\left(g^{(1)}\left(\bar{y}_{\tau}\right) z_{\tau}\right)^{2}\right) .
$$

Here $\beta \geq 0$ is the multiplier associated with the reduced state constraint, and the quadratic form $Q_{1}$ is defined as $Q_{0}$ in (15), but with a costate having a jump

$$
[p(\tau)]=\beta g^{\prime}\left(\bar{y}_{\tau}\right) .
$$

In this way the contribution of the first term in $\Theta^{\prime \prime}(\bar{u})(v, v)$, i.e., $g^{\prime}\left(\bar{y}_{\tau}\right) z_{\tau}^{2}$, is taken into account in $Q_{1}$.

We will only discuss the case when $\beta>0$. By the SONC for the reduced problem, $v=0$ is solution of the problem

$$
\operatorname{Min}_{v \in \mathcal{U}_{2}} Q_{2}(\cdot) ; \quad g^{\prime}\left(\bar{y}_{\tau}\right) z_{\tau} z[v]=0
$$

where the constraint is nothing but $\Theta^{\prime}(\bar{u}) v=0$. The optimality conditions of this problem coincide with (21), with in addition the linearized costate jump that includes the contribution of both the constraint in (88) (with Lagrange multiplier denoted by $\delta \beta$ ) and the expression of $Q_{2}$ :

$$
\left\{\begin{aligned}
{[q(\tau)]=} & \delta \beta g^{\prime}\left(\bar{y}_{\tau}\right)+\beta z_{\tau}^{\top} g^{\prime \prime}\left(\bar{y}_{\tau}\right) \\
& -\beta \gamma^{-1}\left(g_{y}^{(1)}\left(\bar{y}_{\tau}\right) z_{\tau}\right) g_{y}^{(1)}\left(\bar{y}_{\tau}\right) .
\end{aligned}\right.
$$

A second order sufficient condition for local optimality is the existence of $\alpha>0$ such that

$$
Q_{2}(v) \geq \alpha\|v\|_{2}^{2} \quad \text { whenever } g^{\prime}\left(\bar{y}_{\tau}\right) z_{\tau}[v]=0 .
$$

\subsection{Shooting with touch points}

The shooting mapping: $\mathbb{R}^{n+2} \rightarrow \mathbb{R}^{n+2}$ is as follows:

$$
\mathcal{S}:\left(\begin{array}{c}
p_{0} \\
\nu_{1} \\
\tau
\end{array}\right) \mapsto\left(\begin{array}{c}
\phi^{\prime}\left(y_{T}\right)-p_{T} \\
g\left(y_{\tau}\right) \\
g^{(1)}\left(y_{\tau}\right)
\end{array}\right),
$$

where $-[p(\tau)]=\nu_{1} g^{\prime}\left(\bar{y}_{\tau}\right)$.

Theorem 11. Let (79) and (90) hold. Then $\left(\bar{p}_{0}, \nu_{1}, \tau\right)$ is a regular zero of the shooting function.

Proof We just give a sketch of the proof. An analysis similar to the one in the proof of theorem 11 applies, with now $\eta^{1}$ and $\eta^{2}$ equal to zero on both sides, and so locally, $p^{2}$ and $p$ are equal, implying

$$
-[p(\tau)]=\nu_{1} g^{\prime}\left(\bar{y}_{\tau}\right), \text { with } \nu_{1}=[\mu(\tau)] \geq 0 .
$$

Since here $\nu_{2}=\delta \nu_{2}=0$, the relations analogous to (71)(73) reduce to

$$
\left\{\begin{aligned}
-[q(\tau)] & =\nu_{1} z_{\tau}^{\top} g^{\prime \prime}+\lambda^{1} g^{\prime}+\lambda^{2} g_{y}^{(1)} \\
\lambda^{1} & =\delta \nu_{1} ; \quad \lambda^{2}=\delta \tau \nu_{1} .
\end{aligned}\right.
$$


We check as before that an element of the kernel of the shooting mapping corresponds to a stationary point of problem (88). By the above display, we get it whenever

$$
\nu_{1}=-\beta ; \quad \delta \nu_{1}=-\delta \beta ; \quad \delta \tau=-\gamma^{-1} g_{y}^{(1)}\left(\bar{y}_{\tau}\right) z_{\tau} .
$$

We define $\beta$ and $\delta \beta$ so as to satisfy the two first relations. Next, we observe that third one coincides, by (79), with the linearization of the relation $g^{(1)}\left(\bar{y}_{\tau}\right)=0$, and therefore is automatically satisfied. The conclusion follows.

\subsection{Extensions and references}

In the case of multiple state constraints and control variables, an extension of the previous approach is presented in Bonnans and Hermant [2009].

Very often shooting algorithms are used in connection with an homotopy approach: one solves first an easy problem and then tries to follow a path of solution of perturbed problems in order to end with the original one. The structure of the active constraint sets may change. A challenging problem is to analyze these transitions. For scalar first-order state constraints this is relatively wellunderstood: under reasonable hypotheses, a touching point may become inactive, remain as a touching point, or give rise to a boundary arcs and one can give an expansion of its end points, see Bonnans and Hermant [2008].

In the case of a scalar second-order state contraint, Hermant [2010] was able to prove, under weak hypotheses, that a touch point can either be transformed into a boundary arc or two touch points (but no expansion formula is known). An application to the atmospheric reentry problem is provided in Hermant [2011]. So clearly the question of analyzing the transitions between structures of active set constraints is essentially open and challenging. Also there is no analysis yet for the case of touch points at time 0 or $T$.

\section{PROBLEMS WITH SINGULAR ARCS}

\subsection{Framework and shooting algorithm}

We consider the case of a single control: $m=1$, that enters linearly in the state equation, i.e., $f(u, y)=f_{0}(y)+u f_{1}(y)$, so that the state equation reads

$$
\dot{y}_{t}=f_{0}\left(y_{t}\right)+u_{t} f_{1}\left(y_{t}\right), \quad t \in(0, T) ; y_{0}=y^{0},
$$

with control constraint

$$
u_{t} \geq 0, \quad \text { for a.a. } t \in(0, T) .
$$

There is a huge literature on this type of control affine problem, e.g. Schättler and Ledzewicz [2012]. Let $(\bar{u}, \bar{y})$ be a solution and the associated state. The costate equation reads

$$
-\dot{p}_{t}=p_{t} f_{0}^{\prime}\left(\bar{y}_{t}\right)+\bar{u}_{t} p_{t} f_{1}^{\prime}\left(\bar{y}_{t}\right) ; t \in(0, T) ; p_{T}=\phi^{\prime}\left(y_{T}\right) \text {. }
$$

We assume the existence of a switching time $\tau \in(0, T)$, such that the optimal control $\bar{u}$ satisfies

$$
\bar{u}_{t}=0, t \in(0, \tau) ; \quad \bar{u}_{t}>\kappa>0, t \in(\tau, T) .
$$

Let $F$ and $G$ be two smooth vector field in $\mathbb{R}^{n}$. Their Lie bracket is the vector field defined by

$$
[F, G](x):=F^{\prime}(x) G(x)-G^{\prime}(x) F(x), \quad \forall x \in \mathbb{R}^{n} .
$$

Note that $[F, F](x)=0$. We deduce that

$$
\frac{\mathrm{d}}{\mathrm{d} t}\left(p_{t} F\left(\bar{y}_{t}\right)\right)=p_{t}\left[F, f_{0}\right]\left(\bar{y}_{t}\right)+\bar{u}_{t} p_{t}\left[F, f_{1}\right]\left(\bar{y}_{t}\right) \text {. }
$$

Again we define $H(t)$ and related derivatives as in (14). Pontryagin's principle implies that

$$
0=H_{u}(t)=p_{t} f_{1}\left(\bar{y}_{t}\right)=\frac{\mathrm{d}}{\mathrm{d} t}\left(p_{t} f_{1}\left(\bar{y}_{t}\right)\right), \text { over }(\tau, T) \text {. }
$$

So, by $(100)$, since $\left[f_{1}, f_{1}\right]=0$ :

$$
p_{t}\left[f_{1}, f_{0}\right]\left(\bar{y}_{t}\right)=\frac{\mathrm{d}}{\mathrm{d} t}\left(p_{t} f_{1}\left(\bar{y}_{t}\right)\right)=0 \text { over }(\tau, T) .
$$

Applying (100) again, we deduce that, over $(\tau, T)$ :

$$
p_{t}\left[\left[f_{1}, f_{0}\right], f_{0}\right]\left(\bar{y}_{t}\right)+\bar{u}_{t} p_{t}\left[\left[f_{1}, f_{0}\right], f_{1}\right]\left(\bar{y}_{t}\right)=0 .
$$

Assuming, as we will do, that

$$
p_{t}\left[\left[f_{1}, f_{0}\right], f_{1}\right]\left(\bar{y}_{t}\right) \neq 0, \quad t \in[\tau, T],
$$

we deduce an expression of the control as a function of the state and costate, again denoted by

$$
\Upsilon(y, p):=-p\left[\left[f_{1}, f_{0}\right], f_{0}\right](y) / p\left[\left[f_{1}, f_{0}\right], f_{1}\right](y) .
$$

So we can define a shooting algorithm as follows:

$$
\mathcal{S}\left(p_{0}, \tau\right):=\left(\begin{array}{c}
\phi^{\prime}\left(y_{T}\right)-p_{T} \\
p_{T} f_{1}\left(y_{T}\right) \\
p_{T}\left[f_{1}, f_{0}\right]\left(y_{T}\right)
\end{array}\right) .
$$

Here the state-costate system in integrated over $(0, \tau)$ with the control $u_{t}=0$, and over $(\tau, T)$ with $u_{t}=\Upsilon\left(y_{t}, p_{t}\right)$. The two last shooting equations correspond to the two integrations constants so that (103) implies, at a zero of the shooting mapping, that (102) holds.

Note that we have $n+2$ equations and $n+1$ variables. Nevertheless, as proposed in Aronna et al. [2013], we can solve it by minimizing the associated least-square criterion (the norm of the shooting function). Using the GaussNewton algorithm (see e.g. [Bonnans, 2006, Ch. 6]), we obtain local quadratic convergence, provided the derivative of the shooting mapping at the solution is injective. We next analyze this question.

\subsection{The linearized shooting equations}

We have to state the linearization of the shooting equation. As usual we get the linearized state and costate equations. While at time $\tau$ the state and costate are continuous, their derivatives have jumps

$$
[\dot{\bar{y}}(\tau)]=u_{\tau_{+}} f_{1}\left(\bar{y}_{\tau}\right) ; \quad-[\dot{p}(\tau)]=u_{\tau_{+}} p_{\tau} f_{1}^{\prime}\left(y_{\tau}\right) .
$$

Let us set

$$
a:=-\delta \tau u_{\tau_{+}} .
$$

Lemma 1 gives the following jump conditions for the linearized state and costate $(z, q)$ :

$$
\left\{\begin{aligned}
{[z(\tau)] } & =a f_{1}\left(\bar{y}_{\tau}\right) \\
-[q(\tau)] & =a p_{\tau} f_{1}^{\prime}\left(y_{\tau}\right) .
\end{aligned}\right.
$$

The linearization of the shooting equations in (106) gives

$$
\begin{cases}z_{T}^{\top} \phi^{\prime}\left(\bar{y}_{T}\right)-q_{T} & =0, \\ q_{T} f_{1}\left(y_{T}\right)+p_{T} f_{1}^{\prime}\left(y_{T}\right) z_{T} & =0, \\ q_{T}\left[f_{1}, f_{0}\right]\left(y_{T}\right)+p_{T}\left[f_{1}, f_{0}\right]^{\prime}\left(y_{T}\right) z_{T} & =0 .\end{cases}
$$

Next we characterize the linearization of the condition related to the stationarity of the Hamiltonian w.r.t. the control:

Lemma 12. The linearization of the three conditions (103), $\left(p_{T} f_{1}\left(y_{T}\right)\right.$ and $p_{T}\left[f_{1}, f_{0}\right]\left(y_{T}\right)$ is equal to 0 iff

$$
q_{t} f_{1}\left(\bar{y}_{t}\right)+p_{t} f_{1}^{\prime}\left(\bar{y}_{t}\right) z_{t}=0 \text {. a.a. on }(\tau, T) \text {. }
$$


Proof a) We recall the principle of commutation of the operations of time derivation and linearization, see e.g. [Aronna et al., 2013, Lemma 4.1]. That is, if $x_{t}$ belongs to $W^{1, \infty}\left(0, T, \mathbb{R}^{n}\right)$ and $F: \mathbb{R}^{n} \rightarrow \mathbb{R}$, then the linearization is of the time derivative $F^{\prime}\left(x_{t}\right) \dot{x}_{t}$ equal to the derivative of the linearization $F^{\prime}\left(x_{t}\right) \delta x_{t}$ :

$$
F^{\prime \prime}\left(x_{t}\right)\left(\delta x_{t}, \dot{x}_{t}\right)+F^{\prime}\left(x_{t}\right) \delta \dot{x}_{t}=\frac{\mathrm{d}}{\mathrm{d} t}\left(F^{\prime}\left(x_{t}\right) \delta x_{t}\right)
$$

b) The relation

is equivalent to

$$
H_{u}(t):=p_{t} f_{1}\left(\bar{y}_{t}\right)=0, t \in(\tau, T),
$$

$$
H_{u}(T)=\dot{H}_{u}(T)=0 ; \quad \ddot{H}_{u}(t)=0, t \in(\tau, T) .
$$

Similarly, the linearized relation (111) is equivalent to

$$
\left\{\begin{array}{l}
q_{T} f_{1}\left(\bar{y}_{T}\right)+p_{T} f_{1}^{\prime}\left(\bar{y}_{T}\right) z_{T}=0 \\
\frac{\mathrm{d}}{\mathrm{d} t}\left(q_{t} f_{1}\left(\bar{y}_{t}\right)+p_{t} f_{1}^{\prime}\left(\bar{y}_{t}\right) z_{t}\right)_{t=T}=0 \\
\frac{\mathrm{d}^{2}}{\mathrm{~d} t^{2}}\left(q_{t} f_{1}\left(\bar{y}_{t}\right)+p_{t} f_{1}^{\prime}\left(\bar{y}_{t}\right) z_{t}\right)_{t=T}=0, t \in(\tau, T) .
\end{array}\right.
$$

By the above commutation principle it coincides with the linearization of (114). The result follows.

\subsection{A quadratic problem}

Motivated by (109), let us consider the linearized state equation, where $a$ is an optimization variable and $z$ is Lipschitz over $(0, \tau) \cup(\tau, T)$ :

$$
\left\{\begin{array}{l}
\dot{z}_{t}=f^{\prime}\left(\bar{u}_{t}, \bar{y}_{t}\right)\left(v_{t}, z_{t}\right) \\
{[z(\tau)]=a f_{1}\left(\bar{y}_{\tau}\right) ; z_{0}=0 .}
\end{array}\right.
$$

We denote the "average" value of $z$ at time $\tau$ by

$$
\hat{z}=\hat{z}[v, a]=z_{\tau_{-}}+\frac{1}{2} a f_{1}\left(\bar{y}_{\tau}\right),
$$

and consider the following extension of $Q(\cdot)$ defined in (15):

$$
\begin{aligned}
Q(v, a):=\frac{1}{2} \int_{0}^{T} & H^{\prime \prime}(t)\left(v_{t}, z_{t}\right)^{2} \mathrm{~d} t+\frac{1}{2} \phi^{\prime \prime}\left(\bar{y}_{t}\right)\left(z_{T}\right)^{2} \\
& +a p_{\tau} f_{1}^{\prime}\left(\bar{y}_{\tau}\right) \hat{z} .
\end{aligned}
$$

The augmented critical cone is defined as (note that we can take $v \in L^{1}(0, T)$ since $\left.H_{u u}(t)=0\right)$ :

$$
C_{A}(\bar{u}):=\left\{(v, a) \in L^{1}(0, T) \times \mathbb{R} ; \quad v=0 \text { over }(0, \tau)\right\} .
$$

Lemma 13. The quadratic form $Q(v, a)$ is nonnegative over the critical cone.

Proof If $a=0$, then $Q(v, 0)$ is equal to the quadratic from defined in (15), and so is nonnegative by the SONC (36). Next, given an augmented critical direction $(v, a) \in C_{A}(\bar{u})$, for $\sigma>0$, set

$$
v_{t}^{\sigma}= \begin{cases}a / \sigma & \text { over }(\tau, \tau+\sigma), \\ v_{t} & \text { otherwise. }\end{cases}
$$

and $z^{\sigma}:=z\left[v^{\sigma}, 0\right]$. Over $(\tau, \tau+\sigma)$, we have that

$$
\dot{z}_{t}^{\sigma}=\frac{a}{\sigma} f_{1}\left(\bar{y}_{t}\right)+O(1)=\frac{a}{\sigma} f_{1}\left(\bar{y}_{\tau}\right)+O(1),
$$

and so, $z_{t}^{\sigma}=z_{\tau_{-}}+(t-\tau) a \sigma^{-1} f_{1}\left(\bar{y}_{\tau}\right)+O(\sigma)$. It follows that $z\left[v^{\sigma}\right] \rightarrow z[v, a]$ in the space $L^{2}\left(0, T, \mathbb{R}^{n}\right)$, and so,

$$
\int_{\tau}^{\tau+\sigma} v_{t} H_{u y}(t) z_{t}^{\sigma} \mathrm{d} t=\frac{a}{\sigma} \int_{\tau}^{\tau+\sigma} p_{t} f_{1}^{\prime}\left(\bar{y}_{t}\right) z_{t}^{\sigma} \mathrm{d} t
$$

converges to $a p_{\tau} f_{1}^{\prime}\left(\bar{y}_{\tau}\right) z_{\tau}$. Therefore, $Q\left(v^{\sigma}, 0\right) \rightarrow Q(v, a)$. Since $Q\left(v^{\sigma}, 0\right) \geq 0$, the result follows.
Remark 14. This lemma is a particular case of more general results due to Osmolovskiı̌, see Milyutin and Osmolovskiı [1998] and its references, in the more general context when the Hamiltonian is a nonlinear function of the control.

So the problem

$$
\operatorname{Min} Q(v, a) \text { s.t. }(116) \text { and }(v, a) \in C_{A}(\bar{u})
$$

has a cost which is convex over its feasible set which is a vector space. Therefore, its solutions are characterized by the optimality conditions that we express now. The Lagrangian of the problem is

$$
\left\{\begin{aligned}
\mathcal{L}= & Q(v, a)+\int_{0}^{T} q_{t}\left(f^{\prime}\left(\bar{u}_{t}, \bar{y}_{t}\right)\left(v_{t}, z_{t}\right)-\dot{z}_{t}\right) \mathrm{d} t \\
& +r\left(a f_{1}\left(\bar{y}_{\tau}\right)-[z(\tau)]\right) .
\end{aligned}\right.
$$

We expect $q$ to have integrable derivatives on $(0, \tau)$ and $(\tau, T)$, and a jump at time $\tau$. We then get

$$
-\int_{0}^{T} q_{t} \dot{z}_{t} \mathrm{~d} t=\int_{0}^{T} \dot{q}_{t} z_{t} \mathrm{~d} t+[q z(\tau)]-q_{T} z_{T} .
$$

It follows that, taking into account the structure of $f(u, v)$ and hence, of $H(t)$ :

$$
\begin{aligned}
\mathcal{L}_{z} \zeta= & \int_{0}^{T}\left(q_{t} \bar{f}_{y}(t)+\zeta_{t}^{\top} H_{y y}(t)+v p_{t} f_{1}^{\prime}\left(\bar{y}_{t}\right)+\dot{q}_{t}\right) \zeta_{t} \mathrm{~d} t \\
& +\left(z_{T}^{\top} \phi^{\prime \prime}\left(\bar{y}_{T}\right)-q_{T}\right) \zeta_{T} \\
& +\left(r-q_{\tau_{-}}+a p_{\tau} f_{1}^{\prime}\left(\bar{y}_{\tau}\right)\right) \zeta_{\tau_{-}} \\
& +\left(q_{\tau_{+}}-r\right) \zeta_{\tau_{+}}
\end{aligned}
$$

It is easily shown that $\mathcal{L}_{z}=0$ implies that each of the above coefficients is equal to 0 . Eliminating $r=q_{\tau_{+}}$, we obtain the "linearized costate" equation, where $t \in(0, T)$ :

$$
\left\{\begin{aligned}
-\dot{q}_{t} & =q_{t} \bar{f}_{y}(t)+\zeta_{t}^{\top} H_{y y}(t)+v p_{t} f_{1}^{\prime}\left(\bar{y}_{t}\right), \\
q_{T} & =z_{T}^{\top} \phi^{\prime \prime}\left(\bar{y}_{T}\right), \\
-[q(\tau)] & =a p_{\tau} f_{1}^{\prime}\left(\bar{y}_{\tau}\right) .
\end{aligned}\right.
$$

Next we have that

$$
0=\mathcal{L}_{a}=q_{\tau_{+}} f_{1}\left(\bar{y}_{\tau}\right)+p_{\tau} f_{1}^{\prime}\left(\bar{y}_{\tau}\right)\left(z_{\tau_{-}}+a f_{1}\left(\bar{y}_{\tau}\right)\right) .
$$

This is equivalent to

$$
q_{\tau_{+}} f_{1}\left(\bar{y}_{\tau}\right)+p_{\tau} f_{1}^{\prime}\left(\bar{y}_{\tau}\right) z_{\tau_{+}}=0
$$

in which we recognize a linearization "at time $\tau_{+}$" of the relation $H_{u}(t)=p_{t} f_{1}\left(\bar{y}_{t}\right)=0$. Finally the stationarity of the Lagrangian w.r.t. $v$ over $(\tau, T)$ gives

$$
q_{t} f_{1}\left(\bar{y}_{t}\right)+p_{t} f_{1}^{\prime}\left(\bar{y}_{t}\right) z_{t}=0, t \in(\tau, T) .
$$

\subsection{Local study of the shooting mapping}

Consider the following second order condition:

$$
Q(v, a)>0, \text { for any nonzero }(v, a) \in C_{A}(\bar{u}) .
$$

Since, by lemma $13, Q(v, a)$ is nonnegative over the critical cone, this condition is rather weak.

Theorem 15. If (131) holds, then $\left(\bar{p}_{0}, \tau\right)$ is a regular zero of the shooting mapping.

Proof It suffices to observe that, thanks to lemma 12, the linearized shooting equations coincide with the optimality conditions of the problem of minimizing $Q(v, a)$ over the critical cone. Indeed, the first relation (109) correspond to the jump in the state equation, and (127) gives the second one, as well as the final condition on the linearized costate and its dynamics. 
It remains to check the equivalence between the linearization of (103) and the two last relations in (110) on one hand, and (130) on the other (we do not care for (129) since it is implied by (130)). This being consequence of lemma 12, the conclusion follows.

\subsection{References for singular arcs}

The pioneering reference for the analysis of the quadratic conditions is Goh [1966]. Extensions to the case of non unique multipliers (due to e.g. final state constraints) were obtained in Dmitruk [1987] for unconstrained control, and in Aronna et al. [2012] in the case of bounded control and initial-final state constraints. An early reference for the shooting in this context is Maurer [1977]. Another kind of shooting algorithm for problems with singular arcs is presented in Aronna et al. [2013], based on reduction (after some change of time) to the totally singular case (the components of the control are either on one bound, either singular over the all interval $(0, T)$. The version of the present paper is new and more natural, but limited to a simple situation.

Quite often we have mixed singular and non singular control variables, in the sense that the Hamiltonian is affine w.r.t. only some of the control variables. A typical case is the one of the propulsion of an aeroplane; the fuel flow enters linearly in the problem, but in general not the action on the speed direction. Among the studies on the shooting algorithm in this context, see the theoretical work in Aronna [2011], and the application to space launchers in Bonnans et al. [2008] and Martinon et al. [2009].

It may happen that the solution is bang-bang. In that case, covered in Osmolovskii and Maurer [2012], we have a reduction to a finite dimensional optimization problem whose unknowns are the switching times.

\section{BEYOND SHOOTING}

There are some situations where the shooting approach does not apply, but nevertheless, it might be fruitful to adapt some of the ideas of the shooting approach. One example is the class of optimal control problems with distributed delay. In Bonnans et al. [2013] an analysis of the second-order optimality conditions is performed in the presence of state constraints of arbitrary order. In such systems the dynamics depend on the past state and the costate equation has an integral form involving the future states. One can still eliminate the control thanks to an extension of Pontryagins principle. However, the control is then function of the state at the current time, and of the future costates. So, while the shooting approach seems to be meaningless for practical computations, one could try to perform an analysis of switching times in relations with second-order optimality conditions, similar to the one performed in the theory of the classical shooting algorithms.

Similar ideas apply to the optimal control of parabolic equations, and we refer to Lions [1971] as a basic reference on the subject. In the case of a regular Hamiltonian, Bonnans and Jaisson [2010] analyze second-order order optimality conditions (and in fact characterize quadratic growth) and perform a sensitivity analysis, in the presence of first-order state constraints. The Hamiltonian was a strongly convex function of the control. More recently, in Bonnans [2012], a weak form of quadratic growth was characterized in the case of problems with singular arcs. In both cases one can express the control as function of the current state and costate, so that one can reformulate the optimality conditions taking as parameter the initial costate only. Let us detail this in the (simplest) case of a strongly convex Hamiltonian. Let $\Omega$ be an open subset of $\mathbb{R}^{n}$, with say $C^{\infty}$ boundary denoted by $\partial \Omega$. Set $H:=L^{2}(\Omega), V:=H_{0}^{1}(\Omega)$. Consider the control space $\mathcal{U}:=L^{2}(0, T, H)$ and set $Q:=\Omega \times(0, T)$. The state is $y: Q \rightarrow \mathbb{R}$, and the state equation is

$$
\dot{y}-\Delta y=u \text { in } Q
$$

with Dirichlet homogeneous boundary condition $y=0$ over $\partial \Omega \times(0, T)$, and initial condition, where $y_{0}$ is given element in $L^{2}(\Omega)$ :

$$
y(x, 0)=y_{0}(x)
$$

It can be proved, see Lions and Magenes [1972], that the state equation has a unique solution $y[u]$ in the space

$W(0, T):=\left\{y \in L^{2}(0, T, V) ; \dot{y} \in L^{2}\left(0, T, V^{*}\right)\right\}$.

Consider the problem of minimizing

$$
F(u):=\frac{1}{2} \int_{Q} u(x, t)^{2} \mathrm{~d} x \mathrm{~d} t+\frac{1}{2} \int_{\Omega} y[u](x, T)^{2} \mathrm{~d} x .
$$

Then the costate dynamics is

$$
-\dot{p}-\Delta p=0 \text { in } Q
$$

with final condition

$$
p(x, T)=y[u](x, T), \quad x \in \Omega,
$$

and the same Dirichlet homogeneous boundary condition $p=0$ over $\partial \Omega \times(0, T)$. It has a unique solution $p[u] \in W(0, T)$. Let $(\bar{u}, \bar{y}, \bar{p})$ be the unique solution of this problem, and the associated state and costate. Then we can express the control as a function of the costate only:

$$
\bar{u}(x, t)=-\bar{p}(x, t), \quad(x, t) \in Q .
$$

The state-costate dynamics is then

$$
\left\{\begin{aligned}
\dot{\bar{y}}-\Delta \bar{y} & =-\bar{p} \text { in } Q \\
-\dot{\bar{p}}-\Delta \bar{p} & =0 \text { in } Q
\end{aligned}\right.
$$

with boundary conditions

$$
\bar{y}(\cdot, 0)=y_{0} ; \quad \bar{p}(\cdot, T)=y(\cdot, T) .
$$

So the shooting formulation consists in choosing $p_{0}$ in an appropriate subspace of $V$, such that the state-costate dynamics with initial condition $\left(y_{0}, p_{0}\right)$ at time 0 has a unique solution $\left(y\left[p_{0}\right], p\left[p_{0}\right]\right)$. The shooting equation reduces to

$$
p\left[p_{0}\right](\cdot, T)=y\left[p_{0}\right](\cdot, T) .
$$

Since the heat equation has the property of backward uniqueness, Saut and Scheurer [1987], the uniqueness property holds for the function $p\left[p_{0}\right]$, and therefore also for $y\left[p_{0}\right]$. It can be proved that $\bar{y}(\cdot, T)$ belongs to $V$, and so we could take, for the set to which belongs $p_{0}$, the set of initial conditions for the costate equation when the final condition is an arbitrary element of $v$.

In that sense, the shooting formulation is well-defined, even if it is not well-posed in a practical sense (the backward heat equation being unstable). Yet, with a proper functional framework, perhaps one could extend the sensitivity analysis for switching times that is performed in the finite dimensional setting. 


\section{ACKNOWLEDGEMENTS}

Partially supported by the Gaspard Monge Program for Optimization and Operations Research (PGMO) and the ITN-SADCO European project.

\section{REFERENCES}

M.S. Aronna. Singular solutions in optimal control: second order conditions and a shooting algorithm. Rapport de recherche RR-7764, INRIA, October 2011.

M.S. Aronna, J.F. Bonnans, A.V. Dmitruk, and P.A. Lotito. Quadratic order conditions for bang-singular extremals. Numerical Algebra, Control and Optimization, AIMS Journal, 2(3):511-546, 2012.

M.S. Aronna, J.F. Bonnans, and P. Martinon. A wellposed shooting algorithm for optimal control problems with singular arcs. J. Optim. Theory Appl., 2013. Online First, Jan. 2013.

M. Bardi and I. Capuzzo-Dolcetta. Optimal control and viscosity solutions of Hamilton-Jacobi-Bellman equations. Systems and Control: Foundations and Applications. Birkhäuser, Boston, 1997.

J.F. Bonnans. Optimisation Continue. Dunod, Paris, 2006.

J.F. Bonnans. Optimal control of a semilinear parabolic equation with singular arcs. Rapport de recherche RR8099, INRIA, October 2012.

J.F. Bonnans and A. Hermant. Well-posedness of the shooting algorithm for state constrained optimal control problems with a single constraint and control. SIAM J. Control Optim., 46(4):1398-1430, 2007.

J.F. Bonnans and A. Hermant. Stability and sensitivity analysis for optimal control problems with a first-order state constraint and application to continuation methods. ESAIM Control Optim. Calc. Var., 14(4):825-863, 2008.

J.F. Bonnans and A. Hermant. Second-order analysis for optimal control problems with pure state constraints and mixed control-state constraints. Ann. Inst. $H$. Poincaré Anal. Non Linéaire, 26(2):561-598, 2009.

J.F. Bonnans and P. Jaisson. Optimal control of a parabolic equation with time-dependent state constraints. SIAM J. Control Optim, 48(7):4550-4571, 2010.

J.F. Bonnans and J. Laurent-Varin. Computation of order conditions for symplectic partitioned Runge-Kutta schemes with application to optimal control. Numer. Math., 103(1):1-10, 2006.

J.F. Bonnans and A. Shapiro. Perturbation analysis of optimization problems. Springer-Verlag, New York, 2000.

J.F. Bonnans, P. Martinon, and E. Trélat. Singular arcs in the generalized Goddard's problem. J. Optim. Theory Appl., 139(2):439-461, 2008.

J.F. Bonnans, P. Martinon, and V. Grélard. Bocop v1.0.3: A collection of examples. Url: www.bocop.org, June 2012.

J.F. Bonnans, X. Dupuis, and C. de la Vega. First and second order optimality conditions for optimal control problems of state constrained integral equations. $J$. Optim. Theory Applications, March 2013. Online First.

A. E. Bryson, Jr., W. F. Denham, and S. E. Dreyfus. Optimal programming problems with inequality constraints.
I. Necessary conditions for extremal solutions. AIAA J., 1:2544-2550, 1963.

A. Cauchy. Méthode générale pour la résolution des systèmes d'équations simultanées. Comptes Rendus de l'Académie des Sciences de Paris, 27:536-538, 1847.

A.V. Dmitruk. Quadratic conditions for the Pontryagin minimum in an optimal control problem linear with respect to control. II. Theorems on the relaxing of constraints on the equality. Izv. Akad. Nauk SSSR Ser. Mat., 51(4):812-832, 911, 1987.

B.S. Goh. Necessary conditions for singular extremals involving multiple control variables. SIAM J. Control, 4:716-731, 1966.

T. R. Goodman and G. N. Lance. The numerical integration of two-point boundary value problems. Math. Tables Aids Comput., 10:82-86, 1956.

W.W. Hager. Runge-Kutta methods in optimal control and the transformed adjoint system. Numer. Math., 87 (2):247-282, 2000.

A. Hermant. Homotopy algorithm for optimal control problems with a second-order state constraint. Appl. Math. Optim., 61(1):85-127, 2010.

A. Hermant. Optimal control of the atmospheric reentry of a space shuttle by an homotopy method. Optimal Control Appl. Methods, 32(6):627-646, 2011.

D.H. Jacobson, M.M. Lele, and J.L. Speyer. New necessary conditions of optimality for control problems with statevariable inequality constraints. J. Math. Anal. Appl., 35: 255-284, 1971.

K. Jittorntrum. Solution point differentiability without strict complementarity in nonlinear programming. Mathematical Programming, 21:127-138, 1984.

J.-L. Lions. Optimal control of systems governed by partial differential equations. Translated from the French by S. K. Mitter. Die Grundlehren der mathematischen Wissenschaften, Band 170. Springer-Verlag, New York, 1971.

J.-L. Lions and E. Magenes. Non-homogeneous boundary value problems and applications. Vol. I. SpringerVerlag, New York, 1972. Translated from the French by P. Kenneth, Die Grundlehren der mathematischen Wissenschaften, Band 181.

K. Malanowski. Differential stability of solutions to convex, control constrained optimal control problems. Appl. Math. Optim., 12(1):1-14, 1984.

K. Malanowski and H. Maurer. Sensitivity analysis for state constrained optimal control problems. Discrete Contin. Dynam. Systems, 4(2):241-272, 1998.

K. Malanowski, C. Büskens, and H. Maurer. Convergence of approximations to nonlinear optimal control problems. In Mathematical programming with data perturbations, volume 195 of Lecture Notes in Pure and Appl. Math., pages 253-284. Dekker, New York, 1998.

P. Martinon, J.F. Bonnans, J. Laurent-Varin, and E. Trélat. Numerical study of optimal trajectories with singular arcs for an Ariane 5 launcher. J. Guidance, Control, and Dynamics, 32(1):51-55, 2009.

H. Maurer. On optimal control problems with bounded state variables and control appearing linearly. SIAM J. Control Optimization, 15(3):345-362, 1977.

H. Maurer. On the minimum principle for optimal control problems with state constraints. Schriftenreihe des Rechenzentrum 41, Universität Münster, 1979. 
H. Maurer and H.J. Pesch. Solution differentiability for nonlinear parametric control problems. SIAM J. Control Optim., 32(6):1542-1554, 1994.

H. Maurer and H.J. Pesch. Solution differentiability for parametric nonlinear control problems with controlstate constraints. J. Optim. Theory Appl., 86(2):285309, 1995.

A.A. Milyutin and N. P. Osmolovskiu. Calculus of Variations and Optimal Control. American Mathematical Society, Providence, 1998.

D. D. Morrison, J.D. Riley, and J.F. Zancanaro. Multiple shooting method for two-point boundary value problems. Comm. ACM, 5:613-614, 1962.

N.P. Osmolovskii and H. Maurer. Applications to regular and bang-bang control, volume 24 of Advances in Design and Control. Society for Industrial and Applied Mathematics (SIAM), Philadelphia, PA, 2012. Second-order necessary and sufficient optimality conditions in calculus of variations and optimal control.

L.S. Pontryagin, V.G. Boltyanskiǔ, R.V. Gamkrelidze, and E.F. Mishchenko. The mathematical theory of optimal processes. Gordon \& Breach Science Publishers, New York, 1986. Reprint of the 1962 English translation.

J.-C. Saut and B. Scheurer. Unique continuation for some evolution equations. J. of Differential Equations, 66: 118-139, 1987.

H. Schättler and U. Ledzewicz. Geometric optimal control, volume 38 of Interdisciplinary Applied Mathematics. Springer, New York, 2012. Theory, methods and examples.

J. Stoer and R. Bulirsch. Introduction to Numerical Analysis. Springer-Verlag, New-York, 1993. 